\title{
Fetal Macrocephaly: A Novel Sonographic Finding in Congenital Myotonic Dystrophy
}

\author{
Shiri Shinar, MD ${ }^{1}$ Parry Balakumar, BA ${ }^{2}$ Vibhuti Shah, MD ${ }^{3}$ Karen Chong, MD ${ }^{4}$ Tami Uster, MSc ${ }^{4}$ \\ David Chitayat, $\mathrm{MD}^{4,5}$
}

1 Division of Maternal Fetal Medicine, Department of Obstetrics and Gynecology, Ontario Fetal Centre, University of Toronto, Mount Sinai Hospital, Toronto, Ontario, Canada

2 Undergraduate school, McGill University, Montreal, Quebec, Canada

${ }^{3}$ Department of Paediatrics, Mount Sinai Hospital, Toronto, Ontario, Canada

${ }^{4}$ Prenatal Diagnosis and Medical Genetics Program, Department of Obstetrics and Gynecology, University of Toronto, Mount Sinai

Hospital, Toronto, Ontario, Canada

${ }^{5}$ Division of Clinical Genetics and Metabolism, Department of

Pediatrics, University of Toronto, The Hospital for Sickkids, Toronto, Ontario, Canada
Address for correspondence Shiri Shinar, MD, Ontario Fetal Center, Maternal Fetal Medicine, Department of Obstetrics and Gynecology, Mount Sinai Hospital, 700 University Avenue, M5G 1Z5, Toronto, ON, Canada (e-mail: shiri.shinar@sinaihealth.ca).

Am J Perinatol Rep 2020;10:e294-e299.

\begin{abstract}
Keywords

- congenital myotonic dystrophy

- CDM

- macrocephaly

- DM1

- fetal

Objective Sonographic clues to the diagnosis of congenital myotonic dystrophy (CDM) are limited, particularly in the absence of family history of myotonic dystrophy (DM). We reviewed cases of CDM for unique prenatal findings.

Study Design A single-center case series of fetuses with CMD with characteristic prenatal findings confirmed postnatally.

Results Four fetuses with pre- or postnatally diagnosed CDM presented with macrocephaly in utero. While head measurements were appropriate for gestational age until midgestation, third-trimester head circumference and biparietal diameter were both $>2$ standard deviation (SD) above the mean in all. Abdominal and femur measurements were otherwise appropriate for gestation. Postnatally, the occipitofrontal circumference was $>2$ SD above the mean in all, confirming the diagnosis of macrocephaly.

Conclusion CDM should be included in the differential diagnosis of third-trimester macrocephaly, especially in the presence of additional sonographic clues and when maternal medical history and physical examination are suggestive of DM.
\end{abstract}

Myotonic dystrophy type 1 (DM1), an autosomal dominant disorder, is the most commonly inherited neuromuscular disease among adults of European ancestry. ${ }^{1,2}$ Affecting 1 in 7,400 to 10,700 individuals it is also the most common muscular dystrophy among pregnant women. ${ }^{3}$ The clinical expression, though variable, includes progressive muscle weakness, cataract formation, cardiac conduction abnormalities, insulin resistance, temporal baldness, gonadal failure, and infertility. ${ }^{4}$

received

April 21, 2020

accepted

June 4, 2020
DOI https://doi.org/

10.1055/s-0040-1716742. ISSN 2157-6998.
DM1 results from expansion of a cytosine-thymine-guanine (CTG) trinucleotide repeat in the 3 '-untranslated region of the dystrophia myotonica protein kinase (DMPK) gene on chromosome 19q13.3..$^{5}$ An association exists between the number of trinucleotide repeats and disease severity including age of onset. Of its four subtypes, congenital DM (CDM) is the most severe form of the disease, with a widely varying reported incidence, ranging from 2.1 to 28.6 per 100,000 live births. ${ }^{6,7} \mathrm{CDM}$ results predominantly from transmission of

Copyright @ 2020 by Thieme Medical Publishers, Inc., 333 Seventh Avenue, New York, NY 10001, USA. Tel: +1(212) 760-0888.
License terms

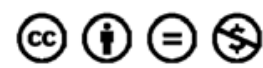


expanded CTG repeats from affected mother-to-child with subsequent worsening of disease severity. ${ }^{8}$ It is characterized by profound hypotonia, myopathic facies, arthrogryposis, poor feeding, and respiratory failure. ${ }^{9}$ Prenatal sonographic findings include polyhydramnios, reduced fetal movements, unior bilateral talipes, and other joint contractures and borderline ventriculomegaly., ${ }^{3,10}$ Despite these sonographic clues, many cases still remain undiagnosed prenatally, particularly when the mother has yet to be diagnosed with DM1.

This case series describes a novel fetal sonographic finding of third-trimester fetal macrocephaly in CDM. This finding can potentially aid in prenatal diagnosis by offering mutation analysis to individuals with a combination of suggestive sonographic features and maternal findings who opt for invasive testing, as well as shed light on the involvement of the DMPK in brain development.

\section{Methods}

A retrospective single-center review of prenatal ultrasounds of fetuses with prenatally or postnatally diagnosed CDM between January 2003 and January 2019. Pregnancies that were terminated or did not have neonatal outcome data were excluded. Second- and third-trimester ultrasounds were reviewed for characteristic sonographic findings. Fetal biometry was assessed according to Hadlock's fetal growth curves ${ }^{11}$ and neonatal biometry was assessed according to the World Health Organization (WHO) child growth standards. ${ }^{12}$ Maternal, pregnancy and neonatal outcomes were reviewed. During this time period, there were there were four cases of CDM that met inclusion criteria. All four presented with thirdtrimester macrocephaly.

This study was approved by our institutional review board.

\section{Results \\ Cases (-Table 1)}

\section{Case 1}

A 24-year-old, G5P2L0 with a known DM1, was followed in our unit during her fifth pregnancy. Her two previous pregnancies had been complicated by CDM and early neonatal deaths. Following the death of her daughter, the patient was diagnosed with DM1. In this pregnancy, she declined invasive testing for CDM. At 34.4 weeks, an ultrasound demonstrated a fetal head circumference (HC) of $333.7 \mathrm{~mm}$ and a biparietal diameter (BPD) of $99 \mathrm{~mm}$ (95th percentile and 99.5th percentile, respectively ${ }^{11}$ ). Additional sonographic findings included polyhydramnios of $32 \mathrm{~cm}$, unilateral severe hydronephrosis, suggestive of ureteropelvic junction stenosis, and absent breathing movements. Delivery was at 38.4 weeks gestation by elective cesarean section. The neonatal occipitofrontal circumference (OFC) was $37 \mathrm{~cm}$ (> 98th percentile, ${ }^{12}$ ). Additional findings included severe hypotonia, myopathic facies, droopy eyelids, tented mouth with a thin upper lip, and absent deep tendon reflexes. Genetic testing confirmed the diagnosis of CDM.

\section{Case 2}

A 21-year-old G2P0 woman was referred to the fetal medicine unit at 35 weeks of gestation, due to the diagnosis of fetal macrocephaly and polyhydramnios. The HC at 35.3 weeks was $345 \mathrm{~mm}$ (98th percentile) and the BPD was $99 \mathrm{~mm}$ (>99th percentile $\left.{ }^{11}\right)$. Additional characteristic findings were mild polyhydramnios and mild unilateral ventriculomegaly of $11 \mathrm{~mm}$. Magnetic resonance imaging (MRI) was attempted but was abandoned due to maternal anxiety. Delivery was by elective cesarean section at 38.3 weeks, due to macrocephaly. The neonatal OFC was $39 \mathrm{~cm}$ ( $>99$ th percentile, $^{12}$ ). Additional neonatal findings included upper lip tenting, severe hypotonia, frontal bossing, down slanting of palpebral fissures, a wide nasal bridge, and weak/absent deep tendon reflexes. Genetic testing confirmed the diagnosis of CDM. Subsequently, the mother was diagnosed with DM1.

\section{Case 3}

A 23-year-old G1P0 woman was followed in our unit due to DM1. She opted for chorionic villus sampling that confirmed the diagnosis of CDM. At 35.4 weeks, the HC measured $357.7 \mathrm{~mm}$ and the BPD was $98.4 \mathrm{~mm}$ (both $>99$ th percentile ${ }^{11}$ ). Additional sonographic findings included moderate polyhydramnios and hydrops fetalis with bilateral pleural effusions, scalp edema, and ascites. Shortly thereafter she had severe preeclampsia and labor was induced, but due to second-stage arrest she had a cesarean section. The neonatal HC was $362 \mathrm{~mm}$ (97th percentile ${ }^{12}$ ). Additional findings included significant whole-body edema, hypotonia, and weak to absent deep tendon reflexes. Resuscitation, intubation, and thoracentesis were undertaken. The infant was admitted to the neonatal intensive care unit and died at 74 days of life secondary to respiratory failure.

\section{Case 4}

A 34-year-old G2P1L1 woman with DM1 was followed in our unit during her second pregnancy. She declined early invasive testing for CDM. At 25 weeks of gestation, she presented with polyhydramnios (amniotic fluid index [AFI]: $32 \mathrm{~cm}$ ) and bilateral talipes. Amniocentesis was performed, confirming the diagnosis of CDM. A repeat ultrasound at 34 weeks demonstrated macrocephaly with an HC of $348.8 \mathrm{~mm}$ and a BPD of $97.1 \mathrm{~mm}$ (both $>99$ th percentile ${ }^{11}$ ). Additional findings included severe polyhydramnios (AFI: $43 \mathrm{~cm}$ ), bilateral hydronephrosis, mild unilateral ventriculomegaly $(10.5 \mathrm{~mm})$, pleural effusions, and minimal ascites. At 35 weeks, due to a biophysical profile of $2 / 8$, she elected for cesarean delivery and postnatal palliative care. The neonate was born alive but died within 30 minutes. Autopsy was declined.

\section{Discussion}

Macrocephaly is a common condition defined as an OFC greater than 2 standard deviation (SD) above and age-related mean, affecting up to $5 \%$ of the pediatric population. In this case series, we describe a novel sonographic finding of fetal macrocephaly in CDM diagnosed in the third trimester of pregnancy despite normal head measurements on earlier 
e296 Macrocephaly in Congenital Myotonic Dystrophy Shinar et al.

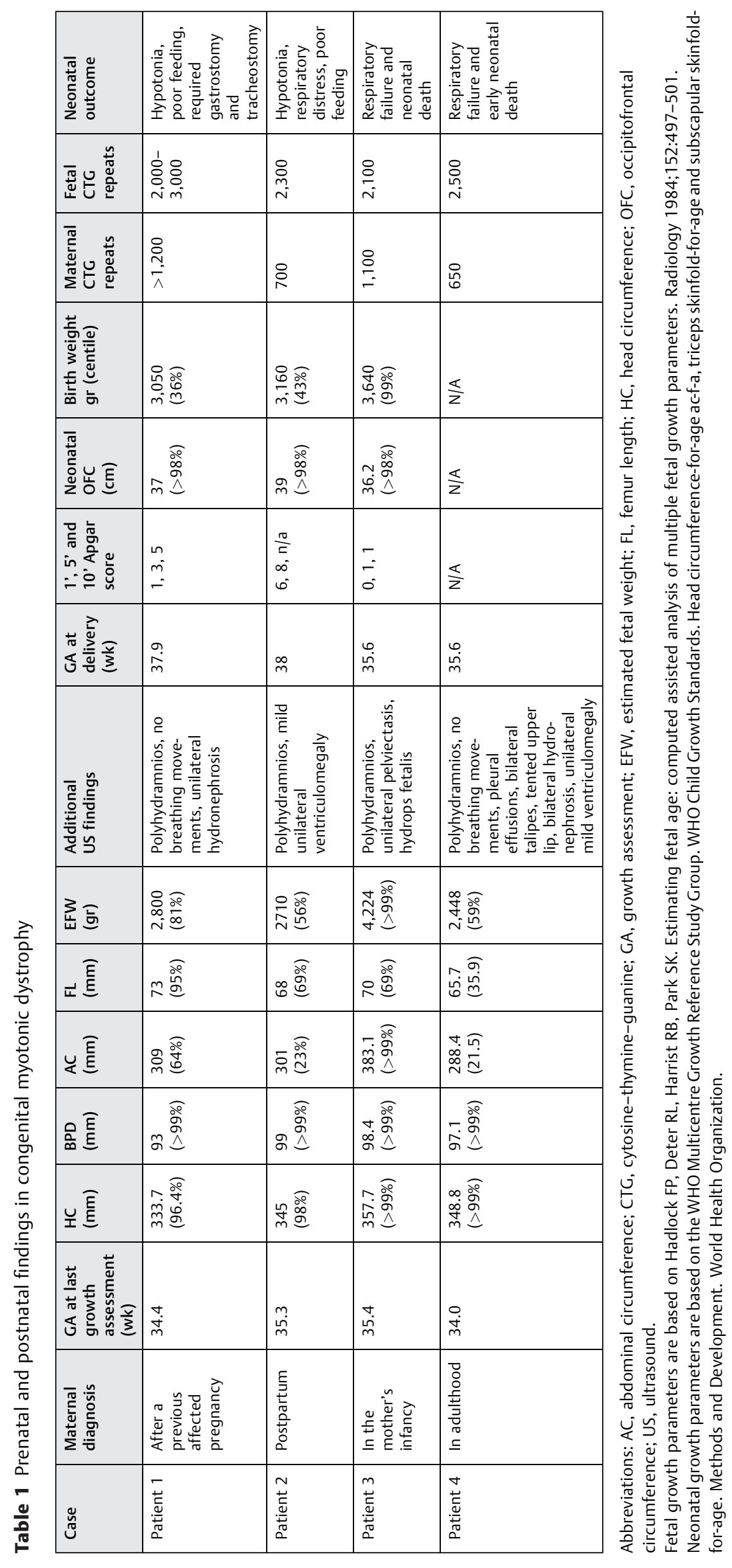

American Journal of Perinatology Reports $\quad$ Vol. $10 \quad$ No. 3/2020 


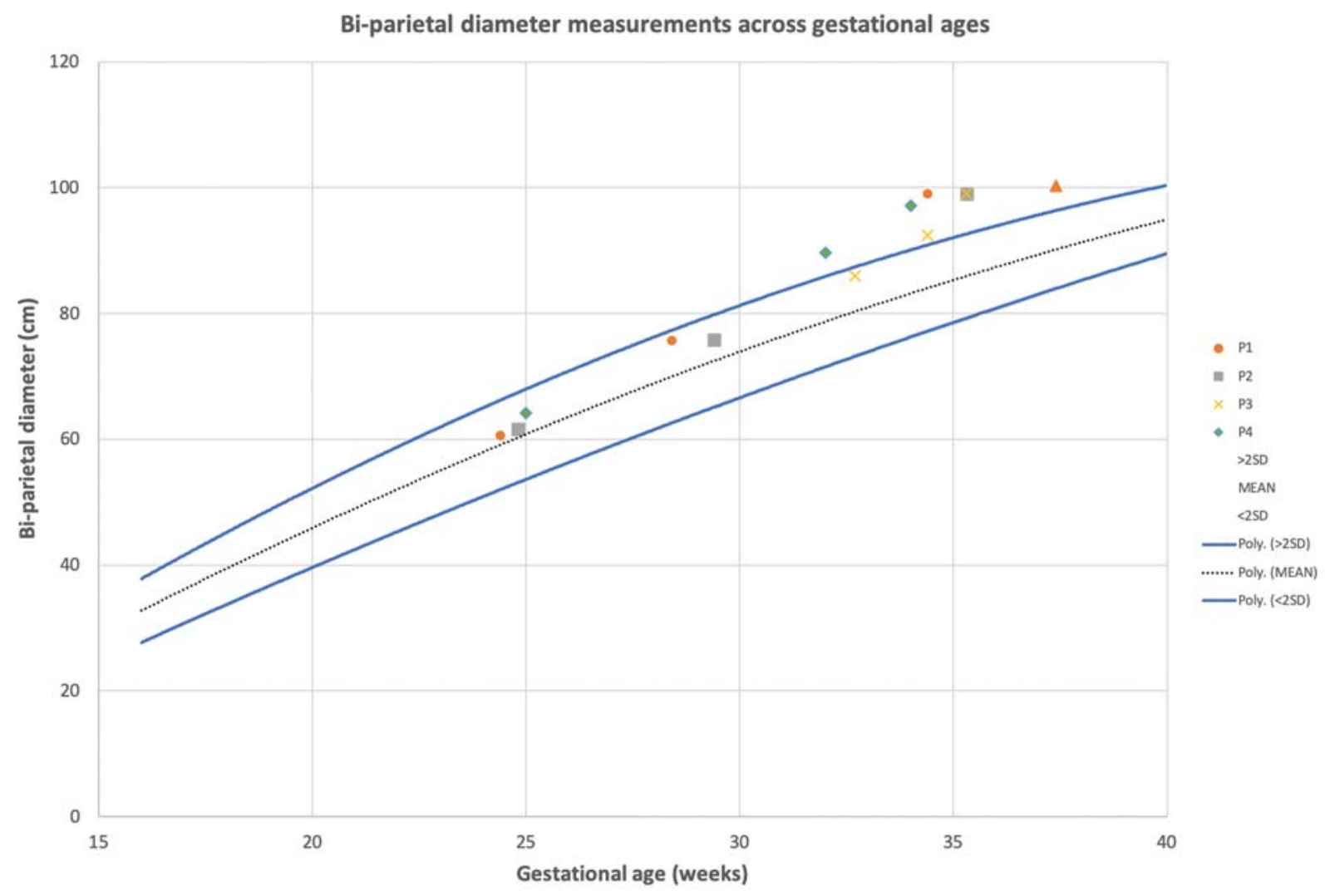

Fig. 1 Biparietal diameter measurements across gestational ages. Dotted line depicts the mean and the solid lines depict two standard deviations (SD) above and below the mean for gestational age according to Hadlock. Adapted from Hadlock FP, Deter RL, Harrist RB, Park SK. Estimating fetal age: computer-assisted analysis of multiple fetal growth parameters. Radiology 1984;152:497-501.

ultrasound scans. In all four fetuses, the BPD and HC were $>2$ SD above the mean (-Figs. 1 and $\mathbf{2}$ ), despite an abdominal circumference (AC), femur length and estimated fetal weight that were appropriate for gestational age. One fetus demonstrated an enlarged AC, but this was secondary to ascites and hydrops ( - Table 1 ). These head measurements along with the borderline to mild ventriculomegaly and the normal subarachnoid space in all cases suggest that CDM may manifest as macrocephaly, possibly due to prenatal megalencephaly. Thus, alterations in DMPK gene may be associated with an enlarged fetal brain.

The association between neonatal mild ventriculomegaly and CDM was first reported by Regev et $\mathrm{al}^{13}{ }^{13}$ who reported this finding in eight of ten neonates with CDM between the age of 1 day and 2 months. In all cases, the HC was within normal range, but head growth was described as excessive in two neonates with intraventricular hemorrhage. Since then sparse incidental reports noted neonatal macrocephaly in cases of CDM. ${ }^{14}$ The first and largest series reporting neonatal macrocephaly in association with CDM was by Garcia-Alix A et al in 1991, who described a series of 14 infants with CDM, ten of whom $(71 \%)$ had macrocephaly. ${ }^{15}$ Eight of these neonates also presented with severe ventriculomegaly. In our series, two of four fetuses (\#2, \#4) presented with mild unilateral ventriculomegaly (defined as an atrial width of $10-12 \mathrm{~mm}$ ), consistent with the ventriculomegaly described in children with $\mathrm{CDM} .{ }^{16} \mathrm{In}$ the other two cases $(\# 1, \# 3)$, the cerebral lateral ventricular sizes were normal. Thus, prenatal macrocephaly in CDM is not secondary to cerebral ventriculomegaly/hydrocephalus, ${ }^{15}$ but instead may arise from an increase in the brain parenchymal volume. While it is plausible that previously described neonates with macrocephaly also demonstrated excessive head growth in utero, to the best of our knowledge, this is the first series to describe fetal macrocephaly in CDM. The third-trimester diagnosis of an enlarged $\mathrm{HC}$ with normal measurements in the second trimester further suggests that accelerating $\mathrm{HC}$ in the third trimester may be a marker for CDM.

Polyhydramnios, a well-known sonographic finding associated with neuromuscular disease, was evident in all four cases. This finding is concordant with a previous large study of 36 cases of prenatally diagnosed CDM that noted polyhydramnios in all affected pregnancies in the late second or early third trimester. ${ }^{10}$ Another commonly described sonographic finding in CDM is uni- or bilateral talipes, ${ }^{10}$ which was detected prenatally in one (\#4) of our four cases.

In the absence of a family history of DM, affected mothers are often only diagnosed after the birth of an affected infant. Indeed, in our series, two cases were detected postnatally with subsequent maternal diagnosis of DM1. Prenatal diagnosis in these cases may be especially challenging if CDM is not considered when nonspecific fetal sonographic findings, such as polyhydramnios, talipes, and mild ventriculomegaly, are detected. Our finding of fetal macrocephaly associated with CDM adds to the few existing clues in cases of lacking 


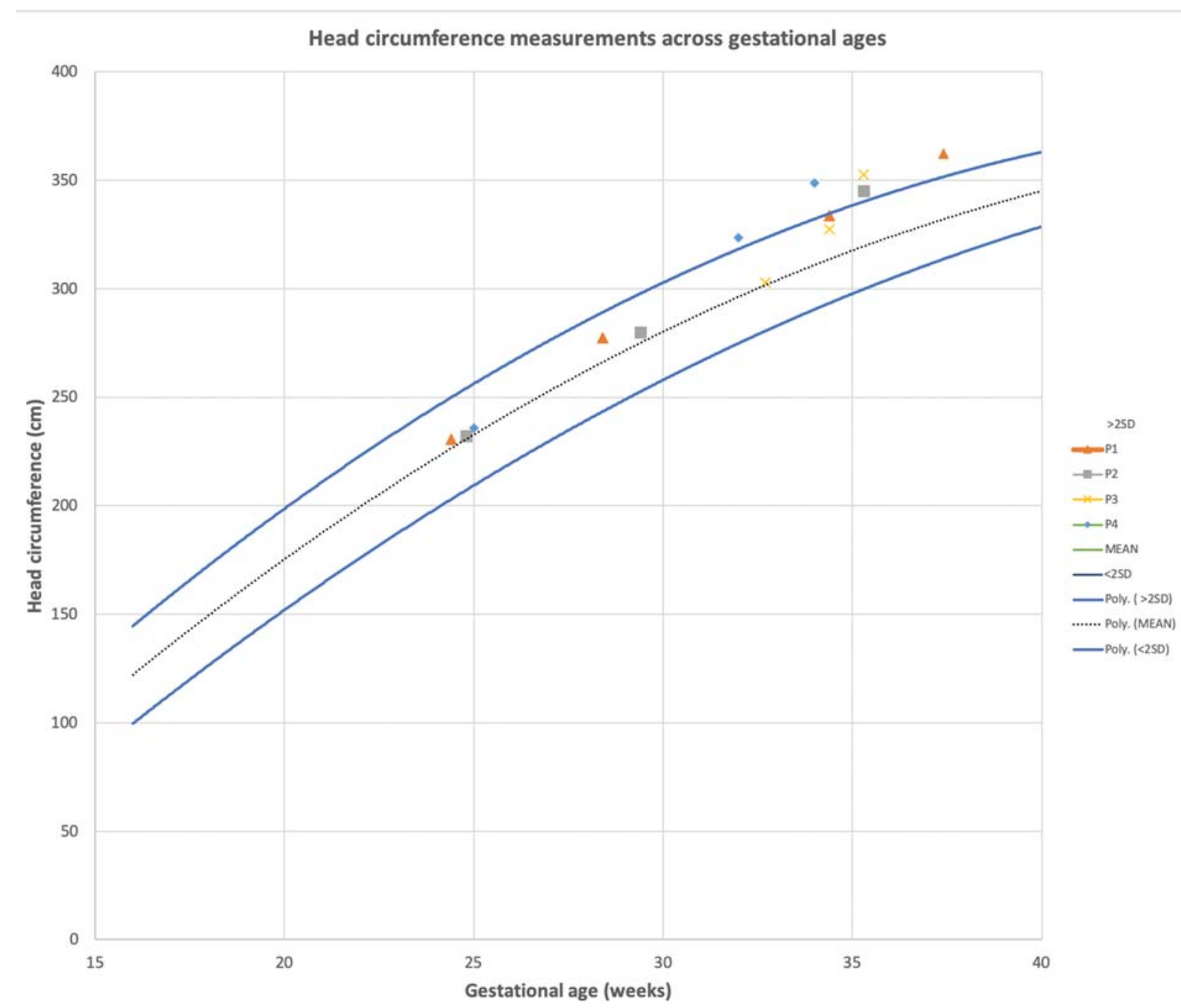

Fig. 2 Head circumference measurements across gestational ages. Dotted line depicts the mean and the solid lines depict two standard deviations (SD) above and below the mean for gestational age according to Hadlock. Adapted from Hadlock FP, Deter RL, Harrist RB, Park SK. Estimating fetal age: computer-assisted analysis of multiple fetal growth parameters. Radiology 1984;152:497-501.

family history DM1/CDM and/or when the mother declines invasive investigation for DM1.

The Fetal Medicine Foundation recommends detailed neurosonography, fetal MRI, and invasive testing for karyotype or chromosomal microarray in cases of fetal macrocephaly. ${ }^{17}$ These evaluations may fail to detect CDM, the diagnosis of which is dependent on maternal examination and specific molecular testing. We recommend considering CDM in the differential diagnosis of third-trimester macrocephaly, especially when parental $\mathrm{HC}$ are within normal limits, maternal history and physical examination are suggestive of DM and additional nonspecific sonographic findings, such as polyhydramnios, are present. Among the maternal signs, grip myotonia is easily tested and pathognomonic to DM, enabling differentiation of CDM from other congenital myopathies. In these cases and in cases where macrocephaly appears along with other well-established sonographic findings suggestive of fetal akinesia/hypokinesia, CDM should be considered and appropriate maternal and fetal testing should be offered.

\section{Data Availability Statement}

The data that support the findings of this study are available on request from the corresponding author. The data are not publicly available due to privacy or ethical restrictions.

\section{Funding}

This research was nonfunded.

\section{Conflict of Interest Statement}

The authors have no conflict of interest to declare.

\section{References}

1 Siciliano G, Manca M, Gennarelli M, et al. Epidemiology of myotonic dystrophy in Italy: re-apprisal after genetic diagnosis. Clin Genet 2001;59(05):344-349

2 Norwood FL, Harling C, Chinnery PF, Eagle M, Bushby K, Straub V. Prevalence of genetic muscle disease in Northern England: indepth analysis of a muscle clinic population. Brain 2009;132(Pt 11):3175-3186

3 Esplin MS, Hallam S, Farrington PF, Nelson L, Byrne J, Ward K. Myotonic dystrophy is a significant cause of idiopathic polyhydramnios. Am J Obstet Gynecol 1998;179(04):974-977 
4 Gutierrez Gutierrez G, Diaz-Manera J, Almendrote M, et al. Clinical guide for the diagnosis and follow-up of myotonic dystrophy type 1, MD1 or Steinert's disease. Neurologia 2020;35(03):185-206

5 Liquori CL, Ricker K, Moseley ML, et al. Myotonic dystrophy type 2 caused by a CCTG expansion in intron 1 of ZNF9. Science 2001;293 (5531):864-867

6 Campbell C, Levin S, Siu VM, Venance S, Jacob P. Congenital myotonic dystrophy: Canadian population-based surveillance study. J Pediatr 2013;163(01):120-5.e1, 3

7 Darin N, Tulinius M. Neuromuscular disorders in childhood: a descriptive epidemiological study from western Sweden. Neuromuscul Disord 2000;10(01):1-9

8 Pratte A, Prévost C, Puymirat J, Mathieu J. Anticipation in myotonic dystrophy type 1 parents with small CTG expansions. Am J Med Genet A 2015;167A(04):708-714

9 Udd B, Krahe R. The myotonic dystrophies: molecular, clinical, and therapeutic challenges. Lancet Neurol 2012;11(10):891-905

10 Zaki M, Boyd PA, Impey L, Roberts A, Chamberlain P. Congenital myotonic dystrophy: prenatal ultrasound findings and pregnancy outcome. Ultrasound Obstet Gynecol 2007;29(03):284-288
11 Hadlock FP, Deter RL, Harrist RB, Park SK. Estimating fetal age: computer-assisted analysis of multiple fetal growth parameters. Radiology 1984;152(02):497-501

12 WHO. WHO Child Growth Standards. Head circumference-for-age ac-f-a, triceps skinfold-for-age and subscapular skinfold-for-age. Methods and Development. Geneva, Switzerland, World Health Organization; 2007

13 Regev R, de Vries LS, Heckmatt JZ, Dubowitz V. Cerebral ventricular dilation in congenital myotonic dystrophy. J Pediatr 1987;111 (03):372-376

14 Bell DB, Smith DW. Myotonic dystrophy in the neonate. J Pediatr 1972;81(01):83-86

15 Garcia-Alix A, Cabañas F, Morales C, et al. Cerebral abnormalities in congenital myotonic dystrophy. Pediatr Neurol 1991;7(01):28-32

16 Mutchnick IS, Thatikunta MA, Gump WC, Stewart DL, Moriarty TM. Congenital myotonic dystrophy: ventriculomegaly and shunt considerations for the pediatric neurosurgeon. Childs Nerv Syst 2016;32(04):609-616

17 https://fetalmedicine.org/education/fetal-abnormalities/brain/ macrocephaly. Accessed July 20, 2020 